\title{
Numerical Calculation of Stresses in Helical Cable Elements subject to Cable Bending and Twisting
}

\author{
Magnus Komperød ${ }^{1}$ \\ ${ }^{1}$ Technological Analyses Centre, Nexans Norway AS, Norway, magnus .komperodenexans .com
}

\begin{abstract}
Calculating the mechanical stresses in cable elements is essential for analyzing the cable's mechanical properties and fatigue properties. This paper derives numerical calculations of the stresses in helical cable elements for cables subject to bending- and twisting loads. The numerical calculations are compared to analytical approximations from the scientific literature. The former gives higher accuracy, and discloses behaviors and coupling effects that are not captured by the latter. These favorable properties combined with easy implementation, no risk of convergence issues, and very short CPU time, make the numerical calculations a very attractive alternative to the analytical approximations.

Keywords: Applied Numerical Analysis; Cross Section Analysis; Differential Geometry; Power Umbilical; Subsea Power Cable; Umbilical.
\end{abstract}

\section{Introduction}

Mechanical analyses of subsea power cables, umbilicals, and power umbilicals establish the relationships between the cables' physical loads and the corresponding mechanical stresses in the cable elements. The physical loads are typically axial cable tension, cable bending curvature, cable twist, and hydrostatic pressure from the surrounding seawater. Physical loads also include the internal pressure inside umbilical tubes. Figure 1 shows an example of an umbilical.

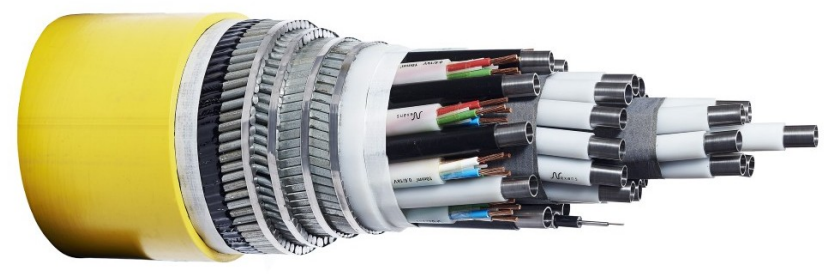

Figure 1. Umbilical with helical tubes, helical electric and fiber optic signal cables, and helical armor wires.

The mechanical cable analyses give essential informa- tion on how the cables will behave during manufacturing, transportation, installation, and operation. Further, the analyses conclude how large physical loads the cable can withstand without the risk of being compromised. Also, the results of these analyses are the basis for subsequent analyses that study the interactions between the cables and their surroundings, including the cables' expected fatigue lives.

Mechanical cable analyses date back to at least half a century ago, with publications written in the 1960s and 1970s still being highly relevant today, such as Lutchansky (1969) and Knapp (1979). Over the later decades the field has matured through improved modeling and by including new considerations in the analyses. Also, some validations against physical testing have been performed, but scientific publications on this topic are unfortunately very sparse. Among the published papers on physical testing are Maioli (2015), Tarnowski (2015), Ekeberg and Dhaigude (2016), Dhaigude and Ekeberg (2016), Jordal et al. (2017), and Komperød et al. (2017).

Like for most other fields of science and engineering, the increased performance and reduced cost of computers over the last decades have strongly influenced mechanical cable analyses. Today, there are several software tools specialized for mechanical analyses of cables and similar structures, such as CableCAD, Helica, UFLEX2D, and UFLEX3D. The author presented a comparison between UFLEX2D simulations and analytical calculations in Komperød (2014).

Also general purpose finite element software tools, such as Abaqus, ANSYS, and COMSOL, are used for analyzing cables' mechanical properties. Several papers have been published on this topic, for example Tjahjanto et al. (2017), which present interesting results on fatigue stresses of helical power phases in a three-phases power cable based on finite element simulations.

Although the field of mechanical cable analyses have matured by the efforts of engineers and scientists over several decades, it is constantly being challenged by the desire of installing and operating cables in ever deeper waters, in lower temperatures, and in harsher weather conditions. This calls for more accurate analyses, for more thorough validation against physical testing, and for inclusion of new considerations in the analyses work, such as cables' sensitivity to the ambient temperature.

The present paper aims to give a contribution to the 
work of improving and extending mechanical cable analyses. The paper derives numerical calculations of the cable elements' stresses and strains subject to cable bending and twisting. Similar results have been presented in the scientific literature using analytical calculations, see for example Kebadze (2000), Sødahl et al. (2010), and Skeie et al. (2012). As such analyses result in difficult mathematical problems, the analytical calculations are simplified, typically through linearizations, until the simplified problem can be solved within reasonable efforts. However, as shown by the author in Komperød (2017), these simplifications give less accurate results and abandon some interesting information.

The work presented in this paper is a continuation of the work presented by the author in Komperød (2017). From the author's point of view, mechanical cable analyses based on numerical solutions of first principle models is an attractive compromise between simplified analytical solutions and complex finite element analyses using advanced software tools. The numerical solution is easy to implement, gives high accuracy, and is efficient in terms of CPU time. The author's preliminary results also indicate that the nature of the numerical calculations make them suitable for parallel processing, which is necessary for taking full advantage of modern computers.

The calculated element stresses will in future works be used to establish the cable's capacity (allowed combinations of axial cable tension and cable bending curvature) and its expected fatigue life. Also, the element stresses and strains give the cable's strain energy, from which the cable's axial stiffness, torsion stiffness, and bending stiffness can be derived.

\section{Nomenclature}

Table 1 presents the nomenclature used in this paper. Subscript is a shorthand notation for discretization steps. For example $f_{-2}$ means $f(x-2 h)$, where the nominal value $x$ is understood from the context. The notation $\|\cdot\|$ means the 2-norm, i.e. the length of a vector.

\section{Assumptions and Simplifications}

The mathematical derivation of this paper is subject to the following assumptions and simplifications:

1. The cable is assumed to have constant bending curvature and constant twist angle along its length.

2. Helical cable elements are assumed to follow a loxodromic curve during cable bending. This means that if the cable element initially follows a thin, helical curve painted on the beneath cable sheath, the cable element will cover the painted curve also after cable bending. Dhaigude and Ekeberg (2016), Ekeberg and Dhaigude (2016), and Tjahjanto et al. (2017) present results from physical tests and finite element simulations which conclude that this assumption holds.
Table 1. Nomenclature.

\begin{tabular}{|c|c|}
\hline$\vec{b}$ & Binormal vector [-]. \\
\hline E & E-modulus [Pa]. \\
\hline$G$ & Shear modulus $[\mathrm{Pa}]$. \\
\hline$h$ & Discretization step [m]. \\
\hline$L$ & Pitch length $[\mathrm{m}]$. \\
\hline$l$ & Cable length parameter [m]. \\
\hline$\vec{n}$ & Normal vector [-]. \\
\hline$\vec{p}$ & $\begin{array}{l}\text { Vector from center of the element to the } \\
\text { point to calculate stresses [m]. }\end{array}$ \\
\hline$p^{\mathrm{r}}$ & Radial component of $\vec{p}[\mathrm{~m}]$ \\
\hline$p^{\mathrm{s}}$ & Surface component of $\vec{p}[\mathrm{~m}]$. \\
\hline$R$ & Pitch radius $[\mathrm{m}]$ \\
\hline$\vec{r}$ & $\begin{array}{l}\text { Parameterization vector of helical cable } \\
\text { element }[\mathrm{m}] \text {. }\end{array}$ \\
\hline$s$ & Cable element length parameter [m]. \\
\hline$\vec{T}$ & Tangent vector [-]. \\
\hline$\vec{t}$ & Unit-length tangent vector [-]. \\
\hline$\vec{u}$ & Unit-length radial vector [-]. \\
\hline$\vec{v}$ & Unit-length surface vector [-]. \\
\hline$\alpha$ & Pitch angle [rad]. \\
\hline$\beta$ & $\begin{array}{l}\text { Helical cable element's angular position } \\
\text { at } l=0[\mathrm{rad}] .\end{array}$ \\
\hline$\gamma_{u t}, \gamma_{v t}, \gamma_{\psi t}$ & Shear strain in helical cable element [-]. \\
\hline$\varepsilon_{t t}$ & Axial strain in helical cable element [-]. \\
\hline$\kappa$ & Cable bending curvature $\left[\mathrm{m}^{-1}\right]$ \\
\hline$\kappa^{\mathrm{h}}$ & $\begin{array}{l}\text { Helical cable element's local curvature } \\
{\left[\mathrm{m}^{-1}\right] \text {. }}\end{array}$ \\
\hline$\vec{\kappa}^{\mathrm{h}}$ & $\begin{array}{l}\text { Helical cable element's local curvature } \\
\text { vector }\left[\mathrm{m}^{-1}\right] .\end{array}$ \\
\hline$\kappa^{\mathrm{r}}$ & Radial component of $\vec{\kappa}^{\mathrm{h}}\left[\mathrm{m}^{-1}\right]$ \\
\hline$\Delta \kappa^{\mathrm{r}}$ & $\begin{array}{l}\text { Difference in } \kappa^{\mathrm{r}} \text { relative to zero-load } \\
{\left[\mathrm{m}^{-1}\right] \text {. }}\end{array}$ \\
\hline$\kappa^{\mathrm{s}}$ & Surface component of $\vec{\kappa}^{\mathrm{h}}\left[\mathrm{m}^{-1}\right]$ \\
\hline$\sigma_{t t}$ & Axial stress in helical cable element [Pa]. \\
\hline$\sigma_{\mathrm{vm}}$ & $\begin{array}{l}\text { von Mises stress in helical cable element } \\
{[\mathrm{Pa}] .}\end{array}$ \\
\hline$\tau_{u t}, \tau_{v t}, \tau_{\psi t}$ & $\begin{array}{l}\text { Shear stress in helical cable element } \\
{[\mathrm{Pa}] \text {. }}\end{array}$ \\
\hline$\varphi$ & Cable twist $[\mathrm{rad} / \mathrm{m}]$ \\
\hline$\varphi^{\mathrm{h}}$ & $\begin{array}{l}\text { Helical cable element's local torsion } \\
{\left[\mathrm{m}^{-1}\right] \text {. }}\end{array}$ \\
\hline$\Delta \varphi^{\mathrm{h}}$ & $\begin{array}{l}\text { Difference in } \varphi^{\mathrm{h}} \text { relative to zero-load } \\
{\left[\mathrm{m}^{-1}\right] \text {. }}\end{array}$ \\
\hline
\end{tabular}

3. The stress calculations assume the helical cable ele- 
ments to follow a linear stress vs. strain relationship.

4. Axial tension and compression in the cable elements are not included in the calculations.

5. Friction / shear forces between cable elements is neglected.

\section{Parameterization of Helical Cable Elements}

The purpose of the present paper is to calculate the stresses and strains of helical cable elements subject to cable bending and cable twisting. These calculations depend on the elements' local curvature and local torsion, which in turn depend on a parameterization of the cable elements' paths in the three-dimensional space.

A parameterization is a function $\mathbb{R} \rightarrow \mathbb{R}^{3}$ which describes a curve in a three dimensional space in terms of a scalar variable. Lutchansky (1969) presents a parameterization of a helical cable element in a bent cable under the assumption that the element follows a loxodromic curve. The author of the present paper provides a similar, but not identical, parameterization in Komperød (2017). The latter parameterization has later been improved by including cable twist. Also, the location of origin of the coordinate system has been improved to give the parameterization better numerical properties. The result is the following parameterization, which will be used in the present paper:

$$
\begin{aligned}
\vec{r}(l)= & {[x(l), y(l), z(l)] } \\
x(l)= & \frac{1}{\kappa}(\cos (\kappa l)-1) \\
& +R \cos \left(\left(\frac{2 \pi}{L}+\varphi\right) l+\beta\right) \cos (\kappa l), \\
y(l)= & R \sin \left(\left(\frac{2 \pi}{L}+\varphi\right) l+\beta\right) \\
z(l)= & {\left[\frac{1}{\kappa}+R \cos \left(\left(\frac{2 \pi}{L}+\varphi\right) l+\beta\right)\right] \sin (\kappa l) . }
\end{aligned}
$$

In Eq. 1, the scalar cable length parameter $l$ expresses the progress of the helical cable element in the threedimensional space. The angle $\beta$ is the element's orientation in the cable cross section at $l=0$. The cable curvature $\kappa$ and the cable twist $\varphi$ are the cable's deformations due to external loads. The pitch length $L$ and the pitch radius $R$ express the helical cable element's geometry relative to the cable's cross section center. The parameterization follows the common convention in the industry: Positive $L$ means right lay-angle of the helical cable element, while negative $L$ means left lay-angle. Usually, successive cable layers have alternating left vs. right lay-angles. The pitch radius $R$ is always positive.
The angles of Eq. 1 have important practical interpretations: The angle $((2 \pi / L+\varphi) l+\beta)$ expresses the helical element's revolution around the cross section center. The angle $\kappa l$ expresses the cable's progress along a virtual circle with radius $1 / \kappa$. The center of the virtual circle is in the point $(-1 / \kappa, 0,0)$.

The parameterization presented in Eq. 1 is not defined for $\kappa=0$, because $\kappa$ occurs in the denominator. The practical interpretation of $\kappa=0$ is that the cable is straight (not bent). Taking the limit $\kappa \rightarrow 0$ gives

$$
\begin{aligned}
\lim _{\kappa \rightarrow 0} \vec{r}(l)= & {\left[\lim _{\kappa \rightarrow 0} x(l), \lim _{\kappa \rightarrow 0} y(l), \lim _{\kappa \rightarrow 0} z(l)\right] } \\
\lim _{\kappa \rightarrow 0} x(l)= & \lim _{\kappa \rightarrow 0} \frac{1}{\kappa}(\cos (\kappa l)-1) \\
& +\lim _{\kappa \rightarrow 0} R \cos \left(\left(\frac{2 \pi}{L}+\varphi\right) l+\beta\right) \cos (\kappa l) \\
= & R \cos \left(\left(\frac{2 \pi}{L}+\varphi\right) l+\beta\right) \\
\lim _{\kappa \rightarrow 0} y(l)= & R \sin \left(\left(\frac{2 \pi}{L}+\varphi\right) l+\beta\right) \\
\lim _{\kappa \rightarrow 0} z(l)= & \lim _{\kappa \rightarrow 0}\left[\frac{1}{\kappa}+R \cos \left(\left(\frac{2 \pi}{L}+\varphi\right) l+\beta\right)\right] \sin (\kappa l) \\
= & l .
\end{aligned}
$$

Evaluating the limits in Eq. 2 results in two zero-overzero expressions, which can easily be evaluated through MacLaurin series expansions of $\cos (\kappa l)$ and $\sin (\kappa l)$, or by using L'Hopital's rule.

The parameterization in Eq. 2 is the parameterization of a helix. This result was expected, because a helical cable element follows a helix curve when the cable is straight. When doing analytical calculations based on the parameterization, the limit $\kappa \rightarrow 0$ can be used. For numerical calculations, however, $\kappa=0$ must be considered a special case, resulting in a parameterization with a piecewise function, i.e. $\vec{r}$ is defined by Eq. 2 for $\kappa=0$, and by Eq. 1 otherwise. Whether very small, but non-zero, absolute values of $\kappa$ may result in numerical difficulties has not been studied by the author and is beyond the scope of this paper.

\section{Calculating Curvature of Helical Cable Element}

This section establishes the relationship between the external cable loads in terms of cable bending curvature, $\kappa$, and cable twist, $\varphi$, and the helical cable element's local curvature vector, $\vec{\kappa}^{\mathrm{h}}$, where the superscript h means "helical element". This vector is essential for subsequent calculation of the element's stress, which is the final goal of this 
paper. The derivation in this section is a brief modification of the author's derivation in Komperød (2017).

The tangent vector $\vec{T}$ of the parameterized curve $\vec{r}$ is defined as

$$
\vec{T} \stackrel{\text { def }}{=} \frac{\mathrm{d} \vec{r}}{\mathrm{~d} l}
$$

and the unit-length tangent vector $\vec{t}$ is defined as

$$
\vec{t} \stackrel{\text { def }}{=} \frac{\vec{T}}{\|\vec{T}\|} .
$$

The definition of curvature from the field of differential geometry is

$$
\kappa \stackrel{\mathrm{h}}{\text { def }}=\left\|\frac{\mathrm{d} \vec{t}}{\mathrm{~d} s}\right\|,
$$

where

$$
\mathrm{d} s \stackrel{\text { def }}{=}\|\mathrm{d} \vec{r}\|
$$

However, for the subsequent calculations of the cable element stresses, it is insufficient to know the curvature as a scalar like it is defined in Eq. 5; also its direction must be known. Hence, for the purpose of mechanical analyses, it is more useful to define the curvature vector as

$$
\vec{\kappa}^{\mathrm{h}} \stackrel{\text { def }}{=} \frac{\mathrm{d} \vec{t}}{\mathrm{~d} s}
$$

Because $\vec{r}$ is parameterized in terms of $l$, not $s$, it is convenient to write the curvature vector as

$$
\begin{aligned}
\vec{\kappa}^{\mathrm{h}} & =\frac{\mathrm{d} l}{\mathrm{~d} s} \frac{\mathrm{d} \vec{t}}{\mathrm{~d} l} \\
& =\frac{1}{\|\vec{T}\|} \frac{\mathrm{d} \vec{t}}{\mathrm{~d} l}
\end{aligned}
$$

where Eqs. 3 and 6 have been used.

A parameterization of the cable's cross section center can be obtained by setting $R=0$ in Eqs. 1 and 2 . In other words; the cross section center is considered as a helical cable element with zero pitch radius. Hence, a unit vector $\vec{u}$ pointing from the physical cable element towards the cable's cross section center can be expressed as

$$
\vec{u} \stackrel{\text { def }}{=} \frac{\left(\left.\vec{r}(l)\right|_{R=0}\right)-\vec{r}(l)}{\left\|\left(\left.\vec{r}(l)\right|_{R=0}\right)-\vec{r}(l)\right\|} .
$$

Taking the dot product between $\vec{T}$ and $\vec{u}$ shows that these vectors are orthogonal. Because $\vec{t}$ and $\vec{T}$ are parallel, $\vec{t}$ and $\vec{u}$ are then orthonormal vectors. Hence, defining $\vec{v}$ as

$$
\vec{v} \stackrel{\text { def }}{=} \vec{t} \times \vec{u}
$$

gives that $\vec{t}, \vec{u}$, and $\vec{v}$ are an orthonormal set of local basis vectors for $\mathbb{R}^{3}$.

As $\vec{t}$ is a unit length vector, its derivative $\mathrm{d} \vec{t} / \mathrm{d} l$ is normal to $\vec{t}$ (please refer to Pressley (2012) p. 11 for proof). Hence, the curvature vector can be expressed as a linear combination of the two other basis vectors. That is

$$
\vec{\kappa}^{\mathrm{h}}=\kappa^{\mathrm{r}} \vec{u}+\kappa^{\mathrm{s}} \vec{v}
$$

for some real scalars $\kappa^{\mathrm{r}}$ and $\kappa^{\mathrm{s}}$. Because $\vec{u}$ and $\vec{v}$ are orthonormal, $\kappa^{\mathrm{r}}$ and $\kappa^{\mathrm{s}}$ are easily found by

$$
\begin{aligned}
\kappa^{\mathrm{r}} & =\vec{\kappa}^{\mathrm{h}} \bullet \vec{u}, \\
\kappa^{\mathrm{s}} & =\vec{\kappa}^{\mathrm{h}} \bullet \vec{v} .
\end{aligned}
$$

Hence, the vector $\left[0, \kappa^{\mathrm{r}}, \kappa^{\mathrm{s}}\right]$ expresses the curvature vector $\vec{\kappa}^{\mathrm{h}}$ in the local Cartesian coordinate system defined by the basis vectors $\vec{t}, \vec{u}$, and $\vec{v}$.

\section{Calculating Torsion of Helical Cable Element}

The curvature derived in the previous section expresses the change in the direction of the tangent vector, $\vec{t}$, of the helical cable element. The physical interpretation is that the element bends locally. The present section considers torsion, which expresses how the two other local basis vectors, $\vec{u}$ and $\vec{v}$, rotate in the plane normal to the tangent vector $\vec{t}$. This interprets into local twisting of the cable element.

The terms "curvature" and "torsion" are used both in the mathematical field of differential geometry and in mechanical cable analyses as in the present paper. Curvature and torsion are defined almost similarly in these two fields, but there is one important difference: Both fields define curvature and torsion based on a local Cartesian coordinate system in which the tangent vector $\vec{t}$ is the first of the three basis vectors. However, differential geometry defines the two other basis vectors as

$$
\begin{aligned}
& \vec{n} \stackrel{\text { def }}{=} \frac{\frac{\mathrm{d} \vec{t}}{\mathrm{~d} s}}{\left\|\frac{\mathrm{d} \vec{t}}{\mathrm{~d} s}\right\|}, \\
& \vec{b} \stackrel{\text { def }}{=} \vec{t} \times \vec{n} .
\end{aligned}
$$

Hence, the difference is that differential geometry defines the second basis vector to be in the direction of the derivative of the tangent vector, while in cable analyses the second basis vector is defined to point in the direction from 
the cable element towards the cable's cross section center. In both fields the third vector is the cross product of the two other vectors.

When the cable is straight (not bent), the helical cable element has the parameterization of a helix. It can be shown that in this case $\vec{n}$ points from the cable element towards the cable's cross section center. Hence, $\vec{n}$ and $\vec{u}$ coincide, which imply that also $\vec{b}$ and $\vec{v}$ coincide.

Differential geometry defines the torsion as

$$
\varphi \stackrel{\text { def }}{=}-\vec{n} \bullet \frac{\mathrm{d} \vec{b}}{\mathrm{~d} s} .
$$

In cable analyses the torsion is defined similarly as

$$
\varphi \stackrel{\text { h def }}{=}-\vec{u} \bullet \frac{\mathrm{d} \vec{v}}{\mathrm{~d} s} .
$$

Again, Eqs. 16 and 17 give the same results if the cable is straight, while the results may be very different when the cable is bent. The definition of Eq. 17 will be used in this paper. that

Because $\vec{u}$ and $\vec{v}$ are orthogonal, i.e. $\vec{u} \bullet \vec{v}=0$, it follows

$$
\begin{aligned}
\frac{\mathrm{d}}{\mathrm{d} s}(\vec{u} \bullet \vec{v}) & =\frac{\mathrm{d} \vec{u}}{\mathrm{~d} s} \bullet \vec{v}+\vec{u} \bullet \frac{\mathrm{d} \vec{v}}{\mathrm{~d} s}=0, \\
\frac{\mathrm{d} \vec{u}}{\mathrm{~d} s} \bullet \vec{v} & =-\vec{u} \bullet \frac{\mathrm{d} \vec{v}}{\mathrm{~d} s} .
\end{aligned}
$$

Hence, the torsion of the helical cable element can alternatively be written as

$$
\varphi^{\mathrm{h}}=\frac{\mathrm{d} \vec{u}}{\mathrm{~d} s} \bullet \vec{v}
$$

Similar as for the curvature, because $\vec{r}$ is parameterized in term of $l$, not $s$, it is convenient to write the torsion as

$$
\begin{aligned}
\varphi^{\mathrm{h}} & =\frac{\mathrm{d} l}{\mathrm{~d} s} \frac{\mathrm{d} \vec{u}}{\mathrm{~d} l} \bullet \vec{v} \\
& =\frac{1}{\|\vec{T}\|} \frac{\mathrm{d} \vec{u}}{\mathrm{~d} l} \bullet \vec{v} .
\end{aligned}
$$

\section{Numerical Calculations}

The two previous sections derive expressions for the curvature and the torsion of a helical cable element. The present section provides numerical calculations to evaluate these expressions. The numerical results will be used in the subsequent calculations of the element's mechanical stresses.

To numerically evaluate the derivatives, the finite difference method is used with a centered, second-order stencil. That is, the derivatives are approximated by

$$
\frac{\mathrm{d} f}{\mathrm{~d} x}=\frac{f_{1}-f_{-1}}{2 h}+\mathscr{O}\left(h^{2}\right) .
$$

In Komperød (2017), the author argued that forth order accuracy is necessary to achieve sufficient accuracy. However, the present paper provides an improved parameterization of the helical cable element's path, $\vec{r}$. This allows smaller discretization steps, $h$, which makes second order accuracy sufficient.

For each value of $l$ to evaluate the curvature and torsion, the follow calculations are performed:

1. The parameterization vector $\vec{r}$ is evaluated for $l-2 h$, $l-h, l, l+h$, and $l+2 h$. That is, $\vec{r}_{-2}, \vec{r}_{-1}, \vec{r}_{0}, \vec{r}_{1}$, and $\vec{r}_{2}$ are calculated based on Eq. 1 and Eq. 2 .

2. The tangent vector $\vec{T}$ is calculated for $l-h, l$, and $l+h$ using the definition of Eq. 3 and the numerical differentiation of Eq. 22. I.e.

$$
\vec{T}_{i}=\frac{\vec{r}_{i+1}-\vec{r}_{i-1}}{2 h}, i \in\{-1,0,1\}
$$

3. The length of the tangent vector, $\|\vec{T}\|$, is calculated for $l-h, l$, and $l+h$.

4. The unit length tangent vector is calculated for $l-h$, $l$, and $l+h$ using Eq. 4. That is,

$$
\vec{t}_{i}=\frac{\vec{T}_{i}}{\left\|\vec{T}_{i}\right\|}, \quad i \in\{-1,0,1\}
$$

5. The curvature vector is calculated at $l$ using Eq. 8 in combination with the numerical differentiation of Eq. 22, i.e.

$$
\vec{\kappa}_{0}^{\mathrm{h}}=\frac{\vec{t}_{1}-\vec{t}_{-1}}{2 h} \frac{1}{\left\|\vec{T}_{0}\right\|}
$$

6. The vector $\vec{u}$ is calculated for $l-h, l$, and $l+h$.

7. The vector $\vec{v}$ is calculated for $l$ using Eq. 10. That is,

$$
\vec{v}_{0}=\vec{t}_{0} \times \vec{u}_{0}
$$

8. The curvature components $\kappa^{\mathrm{r}}$ and $\kappa^{\mathrm{S}}$ are calculated using Eqs. 12 and 13, i.e.

$$
\begin{aligned}
\kappa_{0}^{\mathrm{r}} & =\vec{\kappa}_{0}^{\mathrm{h}} \bullet \vec{u}_{0}, \\
\kappa_{0}^{\mathrm{h}} & =\vec{\kappa}_{0}^{\mathrm{h}} \bullet \vec{v}_{0} .
\end{aligned}
$$


9. The torsion are calculated using Eq. 21 and the numerical differentiation of Eq. 22, which gives

$$
\varphi_{0}^{\mathrm{h}}=\frac{\left(\vec{u}_{1}-\vec{u}_{-1}\right) \bullet \vec{v}_{0}}{2 h} \frac{1}{\left\|\vec{T}_{0}\right\|} .
$$

\section{The Zero-Load-Zero-Stress sumption}

A common assumption in mechanical cable analyses is to assume that all cable elements have zero stress when the cable is subject to no external loads. This means that residual stresses from the production process and other operations are neglected. Hence, the helical cable elements' curvature and torsion when the cable is at rest are used as zero-references for calculating the elements' strains and stresses.

When the cable is at rest, the radial component of the helical cable elements' curvature is

$$
\kappa^{\mathrm{r}}=\frac{4 \pi^{2} R}{4 \pi^{2} R^{2}+L^{2}} .
$$

The surface component of the curvature, $\kappa^{\S}$, is zero when the cable is at rest. The torsion of the helical element is

$$
\varphi^{\mathrm{h}}=\frac{2 \pi L}{4 \pi^{2} R^{2}+L^{2}} .
$$

It simplifies the subsequent calculations to define changes in curvature and torsion relative to the relaxed values as

$$
\begin{aligned}
& \Delta \kappa^{\mathrm{r}}=\kappa^{\mathrm{r}}-\frac{4 \pi^{2} R}{4 \pi^{2} R^{2}+L^{2}}, \\
& \Delta \varphi^{\mathrm{h}}=\varphi^{\mathrm{h}}-\frac{2 \pi L}{4 \pi^{2} R^{2}+L^{2}},
\end{aligned}
$$

where $\kappa^{\mathrm{r}}$ and $\varphi^{\mathrm{h}}$ are the numerical values calculated in the previous section. Because $\kappa^{\mathrm{s}}$ is zero when the cable is at rest, there would be to no avail to introduce a corresponding notation for this variable.

\section{Analytical Approximations from the Scientific Literature}

The author is not familiar with other scientific publications that calculate helical cable elements' curvature and torsion numerically, except for the author's own paper Komperød (2017). However, there are several publications on analytical approximations of these values. It is then of interest to compare the analytical and numerical approaches. The analytical approximations of Skeie et al. (2012) and Kebadze (2000) will be used for comparison in this paper.
Skeie et al. (2012) and Kebadze (2000) use the pitch angle, $\alpha$, instead of the pitch length, $L$. The pitch angle is defined as

$$
\alpha=\arctan \left(\frac{2 \pi R}{L}\right)
$$

The derivation of Skeie et al. (2012) results in these formulas

$$
\begin{aligned}
\Delta \kappa^{\mathrm{r}}= & \cos ^{4}(\alpha) \cos \left(\frac{2 \pi}{L} l+\beta\right) \kappa \\
& +2 \cos ^{3}(\alpha) \sin (\alpha) \varphi \\
\kappa^{\mathrm{s}}= & -\cos (\alpha)\left(1+\sin ^{2}(\alpha)\right) \sin \left(\frac{2 \pi}{L} l+\beta\right) \kappa \\
\Delta \varphi^{\mathrm{h}}= & -\cos ^{3}(\alpha) \sin (\alpha) \cos \left(\frac{2 \pi}{L} l+\beta\right) \kappa \\
& +\cos ^{2}(\alpha) \cos (2 \alpha) \varphi
\end{aligned}
$$

Kebadze (2000) provides these formulas

$$
\begin{aligned}
\Delta \kappa^{\mathrm{r}}= & \cos (\alpha) \cos \left(\frac{2 \pi}{L} l+\beta\right) \kappa \\
& +2 \cos ^{3}(\alpha) \sin (\alpha) \varphi, \\
\kappa^{\mathrm{s}}= & -\sin \left(\frac{2 \pi}{L} l+\beta\right) \kappa, \\
\Delta \varphi^{\mathrm{h}}= & -\sin (\alpha) \cos \left(\frac{2 \pi}{L} l+\beta\right) \kappa \\
& +\cos ^{2}(\alpha) \sin ^{4}(\alpha) \varphi .
\end{aligned}
$$

Eqs. 35-40 are adapted to fit the notation and the orientation of the helical element used in this paper.

\section{Example 1}

This section compares the numerical calculations derived in this paper with the formulas of Skeie et al. (2012) and Kebadze (2000). The geometry of the helical cable element used in the example is given in Table 2.

Table 2. Geometry of helical cable element used in Examples 1 and 2 .

\begin{tabular}{lr}
\hline Property & Value \\
\hline Pitch length, $L[\mathrm{~m}]$ & 5.0 \\
Pitch radius, $R[\mathrm{~m}]$ & 0.20 \\
Initial angle, $\beta[\mathrm{rad}]$ & 0.00 \\
\hline
\end{tabular}

The analytical and numerical approaches will be compared for the three load scenarios given in Table 3. Hence, 
the first scenario is twist only, the second scenario is curvature only, while the third scenario is both curvature and twist. Figures 2, 3, and 4 show the curvatures $\Delta \kappa^{\mathrm{r}}$ and $\kappa^{\mathrm{s}}$, and the twist $\Delta \varphi^{\mathrm{h}}$, plotted against the cable length parameter $l$ for load scenarios \#1, \#2, and \#3, respectively.

Table 3. Load scenarios used in Examples 1 and 2.

\begin{tabular}{lrr}
\hline Scenario & $\kappa\left[\mathrm{m}^{-1}\right]$ & $\varphi[\mathrm{rad} / \mathrm{m}]$ \\
\hline$\# 1$ & 0.00 & $3.49 \times 10^{-2}$ \\
$\# 2$ & 0.20 & $0.00 \times 10^{-2}$ \\
$\# 3$ & 0.20 & $3.49 \times 10^{-2}$ \\
\hline
\end{tabular}
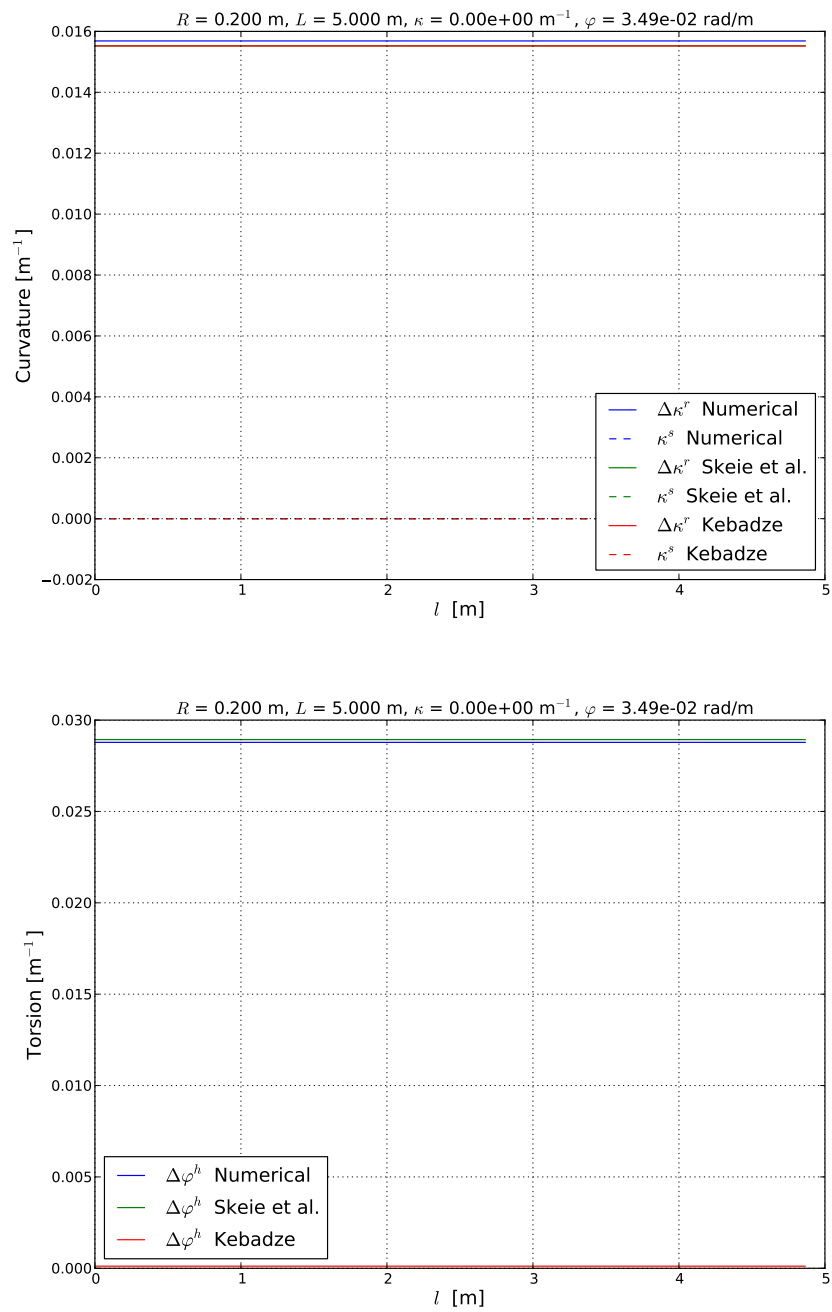

Figure 2. Curvatures $\Delta \kappa^{\mathrm{r}}$ (upper), $\kappa^{\mathrm{s}}$ (upper), and torsion $\Delta \varphi^{\mathrm{h}}$ (lower) for load scenario \#1 given by Tables 2 and 3 .

Figure 2 represents load scenario 1 , which is the twistonly case. The figure shows that twisting the cable adds offsets to $\Delta \kappa^{\mathrm{r}}$ and $\Delta \varphi^{\mathrm{h}}$, while the graphs remains horizontal, straight lines. The curvature $\kappa^{\mathrm{s}}$ does not change at all, i.e. it remains at zero. These results are expected, because after twisting the cable, the helical elements still have the
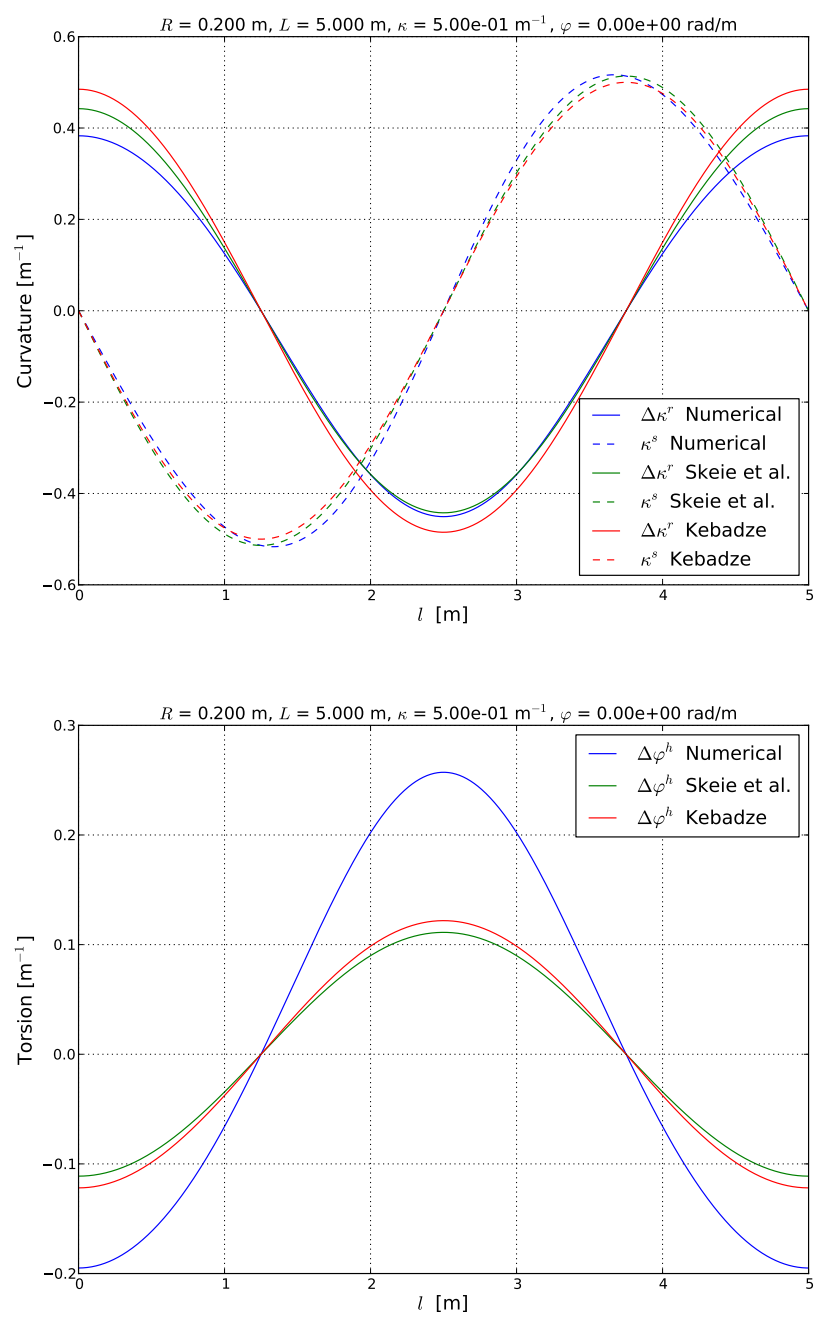

Figure 3. Curvatures $\Delta \kappa^{\mathrm{r}}$ (upper), $\kappa^{\mathrm{s}}$ (upper), and torsion $\Delta \varphi^{\mathrm{h}}$ (lower) for load scenario \#2 given by Tables 2 and 3.

shapes of helices, which are known to have constant $\kappa^{\mathrm{r}}$ and $\varphi^{\mathrm{h}}$ over the length $l$, and zero $\kappa^{\mathrm{s}}$.

For load scenario \#1 the numerical calculation derived in this paper and the approximation of Skeie et al. (2012) are similar to the extent that they can hardly be distinguished to the resolution of Figure 2. Kebadze (2000) give the same result for $\kappa^{\mathrm{s}}$ as the two other approaches, and also very similar result for $\Delta \kappa^{\mathrm{r}}$. For $\Delta \varphi^{\mathrm{h}}$, Kebadze (2000) gives very different results. This can also be seen by comparing the second row of Eq. 37 and the second row of Eq. 40. The latter has the factor $\sin ^{4}(\alpha)$ which is very small for realistic pitch angles. It is not known to the author whether this is Kebadze's actual result or whether it is a typo in an otherwise excellent $\mathrm{PhD}$ thesis.

Load scenario \#2, i.e. the curvature-only case, is shown in Figure 3. While twist adds offsets to the graphs, curvature induces sinusoidal-like oscillations around zero for $\Delta \kappa^{\mathrm{r}}, \kappa^{\mathrm{s}}$, and $\Delta \varphi^{\mathrm{h}}$. As seen from Eqs. 35-40, the formulas of Skeie et al. (2012) and Kebadze (2000) are perfect sinusoidals, while Figure 3 shows that the numerical ap- 

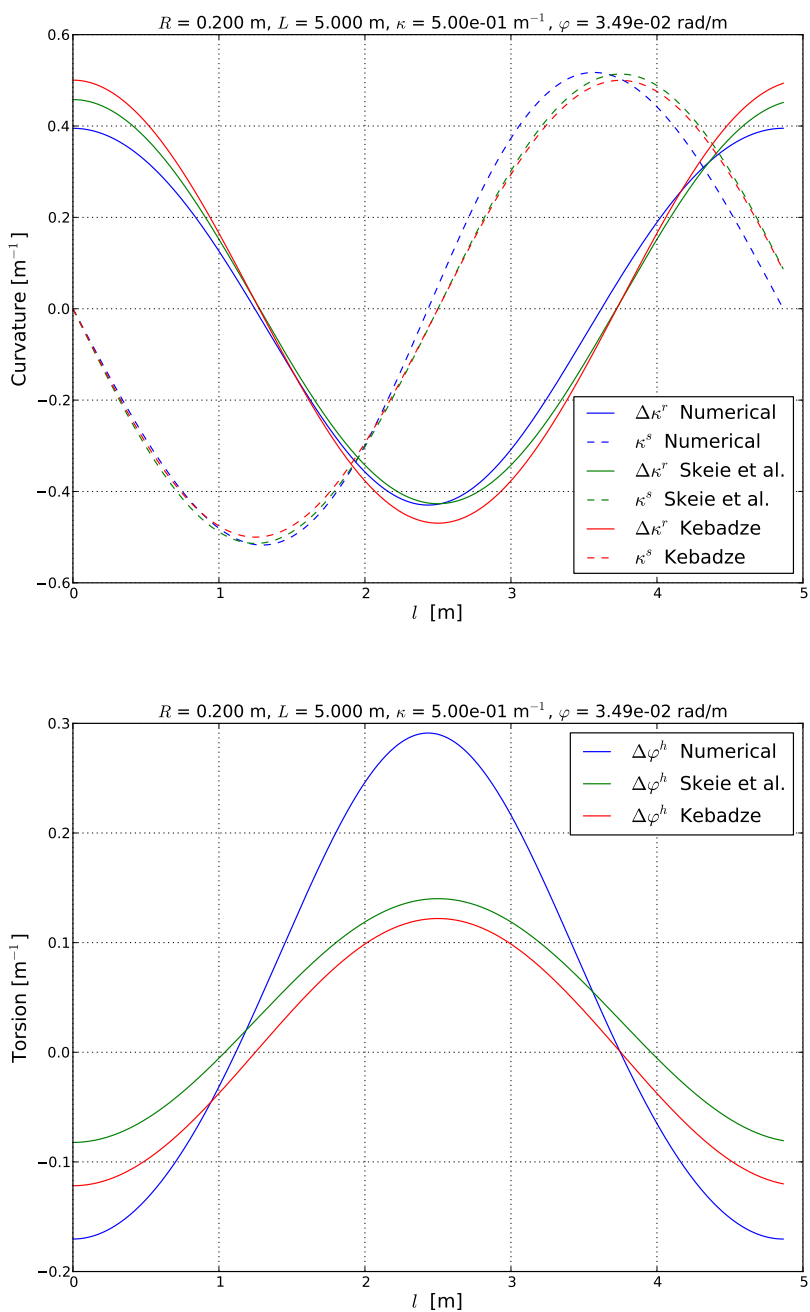

Figure 4. Curvatures $\Delta \kappa^{\mathrm{r}}$ (upper), $\kappa^{\mathrm{s}}$ (upper), and torsion $\Delta \varphi^{\mathrm{h}}$ (lower) for load scenario \#3 given by Tables 2 and 3 .

proach produces graphs that differ somewhat from true sinusoidals.

The most distinct observation in Figure 3 is the difference in amplitude for $\Delta \varphi^{\mathrm{h}}$ between the numerical calculation and the two analytical approximations. The former gives an amplitude that is roughly twice as large as the latter two. To the author's understanding, the difference is caused by an oversimplification in the analytical approximations: Skeie et al. (2012) parameterize the helical cable element in $s$, which is defined by Eq. 6, but simplify by introducing an approximation equivalent to

$$
s=\frac{l}{\cos (\alpha)},
$$

which is exact only for $\kappa=0$. In words this means that the analytical approximation neglects that the element is elongated in the cable's outer arc of bending and compressed in the inner arc. In the notation of the present paper this corresponds to simplifying Eq. 4 to

$$
\vec{t}=\vec{T} \cos (\alpha)
$$

After doing the same simplification in the numerical calculation, the result of load scenario \#2 becomes as shown in Figure 5. As seen from the figure, there is then quite good agreement between the numerical calculation and the analytical approximations. Hence, this comparison supports that the difference in the lower subplot of Figure 3 is caused by the oversimplification in the analytical approximations.

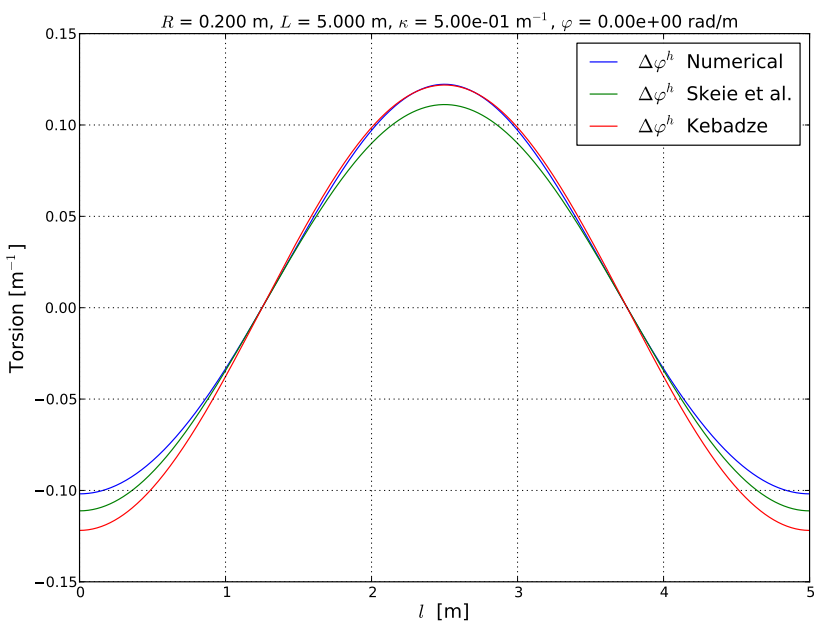

Figure 5. Torsion $\Delta \varphi^{\mathrm{h}}$ for load scenario \#2 given after simplifying Eq. 4 to Eq. 42 .

Load scenario \#3, which is shown in Figure 4, demonstrates a coupling effect between the cable bending curvature, $\kappa$, and the cable twist, $\varphi$, which is only captured by the numerical calculation: When the cable is twisted, the curvatures and the torsion are still periodic, but with a period that is somewhat different from $L$, i.e. somewhat different from $5.0 \mathrm{~m}$ in the figure. In other words; twisting the cable increases or decreases the spatial frequency of the curvature oscillations and the torsion oscillation. The analytical approximations inherently fail to include this effect, because they are linearized in both $\kappa$ and $\varphi$.

\section{Calculating Mechanical Stresses}

The previous sections derive numerical calculations of local curvature and local torsion of helical cable elements, and show that there are some differences between the numerical calculations and the analytical approximations presented by Skeie et al. (2012) and by Kebadze (2000). The present section shows how to calculate the elements' strains and stresses from the local curvature and the local torsion. There is full agreement between the numerical approach and the analytical approaches for how to calculate the strains and the stresses, once the curvature and the torsion have been established. 
The problem of interest is to calculate the axial strain, $\varepsilon_{t t}$, the axial stress, $\sigma_{t t}$, the shear strains, $\gamma_{u t}$ and $\gamma_{v t}$, the shear stresses, $\tau_{u t}$ and $\tau_{v t}$, and the von Mises stress, $\sigma_{\mathrm{vm}}$, at a specified point in the helical cable element's cross section. Let $\vec{p}$ be a vector from the helical cable element's cross section center to the specified point defined in the local orthonormal basis $\vec{t}, \vec{u}$, and $\vec{v}$. Because $\vec{t}$ is normal to the element's cross section plane, while $\vec{p}$ lies in this plane, $\vec{p}$ has at most two nonzero components. Hence, $\vec{p}$ is on the form

$$
\vec{p}=\left[0, p^{\mathrm{r}}, p^{\mathrm{s}}\right]
$$

The axial strain, $\varepsilon_{t t}$, is the negative dot product between the curvature vector and the $\vec{p}$ vector where both are in reference to the orthonormal basis $\vec{t}, \vec{u}$, and $\vec{v}$, i.e.

$$
\begin{aligned}
\varepsilon_{t t} & =-\left[0, \Delta \kappa^{\mathrm{r}}, \kappa^{\mathrm{s}}\right] \bullet\left[0, p^{\mathrm{r}}, p^{\mathrm{s}}\right] \\
& =-\Delta \kappa^{\mathrm{r}} p^{\mathrm{r}}-\kappa^{\mathrm{s}} p^{\mathrm{s}}
\end{aligned}
$$

The axial stress, $\sigma_{t t}$, is the axial strain multiplied by the E-modulus, which gives

$$
\begin{aligned}
\sigma_{t t} & =E \varepsilon_{t t} \\
& =-E\left(\Delta \kappa^{\mathrm{r}} p^{\mathrm{r}}+\kappa^{\mathrm{s}} p^{\mathrm{s}}\right) .
\end{aligned}
$$

The shear strains are calculated as

$$
\begin{aligned}
& \gamma_{u t}=-\Delta \varphi^{\mathrm{h}} p^{\mathrm{s}}, \\
& \gamma_{v t}=\Delta \varphi^{\mathrm{h}} p^{\mathrm{r}} .
\end{aligned}
$$

The corresponding shear stresses are then

$$
\begin{aligned}
\tau_{u t} & =G \gamma_{u t} \\
& =-G \Delta \varphi^{\mathrm{h}} p^{\mathrm{s}}, \\
\tau_{v t} & =G \gamma_{v t} \\
& =G \Delta \varphi^{\mathrm{h}} p^{\mathrm{r}} .
\end{aligned}
$$

In mechanical cable analyses, it is often not necessary to know $\gamma_{u t}$ and $\gamma_{v t}$ as individual components. This leads to an alternative approach: Replace the local Cartesian coordinate system $\vec{t}, \vec{u}$, and $\vec{v}$ with a cylindrical coordinate system, where $\vec{t}$ is the cylinder's length direction, and $\vec{u}$, and $\vec{v}$ are replaced by polar coordinates. The shear stress necessary for the analyses can then be expressed by

$$
\gamma_{\psi t}=\Delta \varphi^{\mathrm{h}} \sqrt{p^{\mathrm{r} 2}+p^{\mathrm{s} 2}}
$$

where $\psi$ is the argument of the polar coordinate system and the square root expression is its magnitude. The stress is then

$$
\begin{aligned}
\tau_{\psi t} & =G \gamma_{\psi t} \\
& =G \Delta \varphi^{\mathrm{h}} \sqrt{p^{\mathrm{r} 2}+p^{\mathrm{s} 2}} .
\end{aligned}
$$

The format of Eqs. 50 and 51 is suitable for calculating the von Mises stress and the strain energy.

The axial stress and the shear stresses discussed in this section gives the follwing expression for the von Mises stress

$$
\sigma_{\mathrm{vm}}=\sqrt{\sigma_{t t}^{2}+3 \tau_{\psi t}^{2}}
$$

where $\sigma_{t t}$ and $\tau_{\psi t}$ are given by Eqs. 45 and 51, respectively.

\section{Example 2}

This example continues Example 1 by calculating stresses as derived in the previous section for the geometry given in Table 2 and the load scenarios given in Table 3 . In addition to these data, the helical element's cross section geometry and material properties must be given. Assume a $10 \times 3$ $\mathrm{mm}$ rectangular steel armor wire, where the stresses at the corner in the first quadrant, i.e. along the positive $\vec{u}$ and the positive $\vec{v}$, are to be calculated. The $\vec{p}$ vector and the material properties are as given in Table 4.

Table 4. The $\vec{p}$ vector and the material properties used in Example 2.

\begin{tabular}{lr}
\hline Property & Value \\
\hline$p^{\mathrm{r}}[\mathrm{m}]$ & $1.5 \times 10^{-3}$ \\
$p^{\mathrm{s}}[\mathrm{m}]$ & $5.0 \times 10^{-3}$ \\
E-modulus, $E[\mathrm{~Pa}]$ & $2.0 \times 10^{11}$ \\
Shear modulus, $G[\mathrm{~Pa}]$ & $7.5 \times 10^{10}$ \\
\hline
\end{tabular}

Figure 6 shows the calculated stress for load scenarios \#1 (upper), \#2 (middle), and \#3 (lower). As expected from Example 1, the axial stress and the shear stress are quite similar to sinusoidals when the cable is bent, and straight lines otherwise.

\section{Further Work}

The results presented in this paper are part of a larger work to develop a framework for numerical analyses of cables' and umbilicals' mechanical properties and fatigue properties. The next step is to develop numerical calculations for cables' axial stiffness, torsion stiffness, and bending stiffness. Developing the numerical analyses is part of Nexans Norway's continuous work on improving the accuracy of 


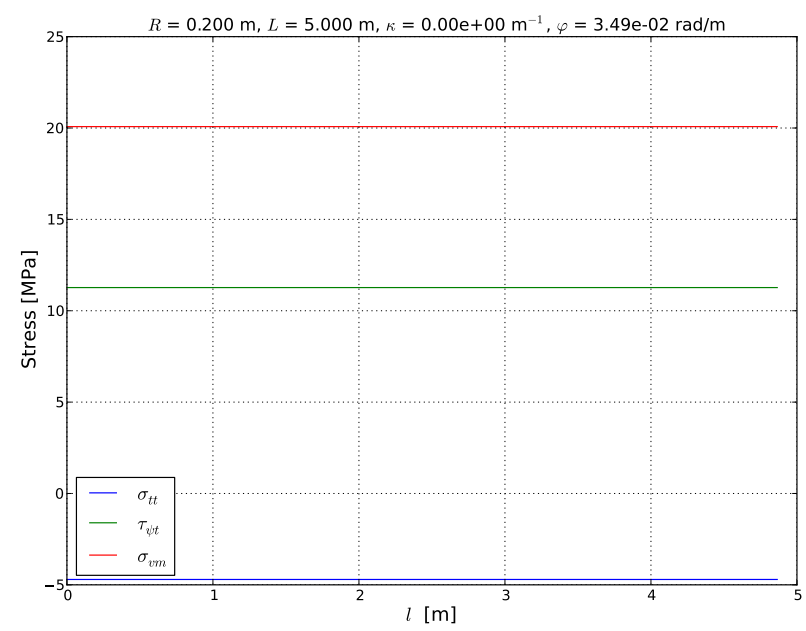

weather conditions.

\section{Conclusion}

This paper derives numerical calculations of local curvature, local torsion, axial stress, shear stress, and von Mises stress for helical elements in cables and umbilicals subject to bending loads and twisting loads.

The numerical calculations disclose behaviors that analytical approximations from the scientific literature fail to identify. The numerical calculations also identified an erroneous approximation done by the analytical approaches, which probably is caused by an oversimplification.

The numerical calculations are simple to implement and do not depend on any iteration process that could cause convergence issues. The calculations are also very fast in terms of CPU time. From the author's point of view,

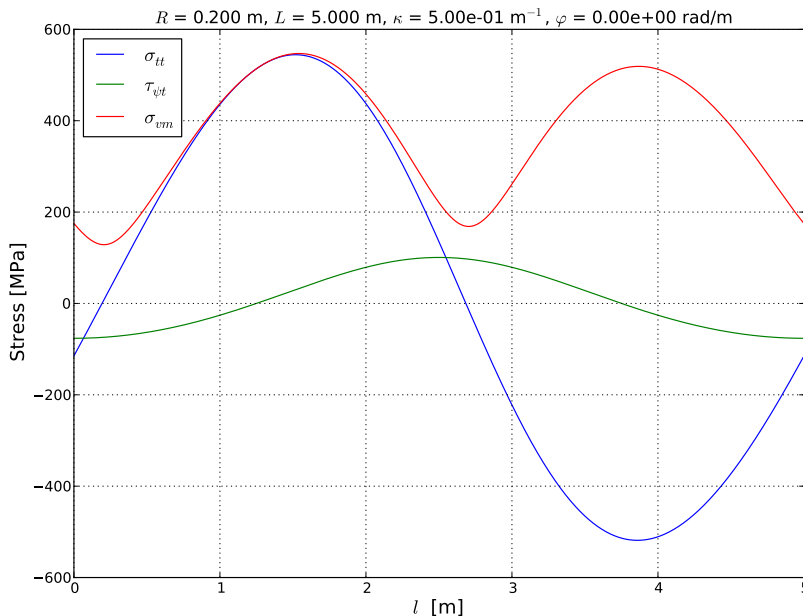
the numerical approach is a very attractive alternative to the analytical approximations, because it gives increased accuracy and deeper insight without any significant disadvantages.

\section{References}

M. M. Dhaigude and K. I. Ekeberg. Validation of the loxodromic bending assumption using high-quality stress measurements - high tension case. In Proceedings of the Twenty-sixth International Ocean and Polar Engineering Conference (ISOPE 2016), Rhodes, Greece, 2016.

K. I. Ekeberg and M. M. Dhaigude. Validation of the loxodromic bending assumption using high-quality stress measurements. In Proceedings of the Twenty-sixth International Ocean and Polar Engineering Conference (ISOPE 2016), Rhodes, Greece, 2016.

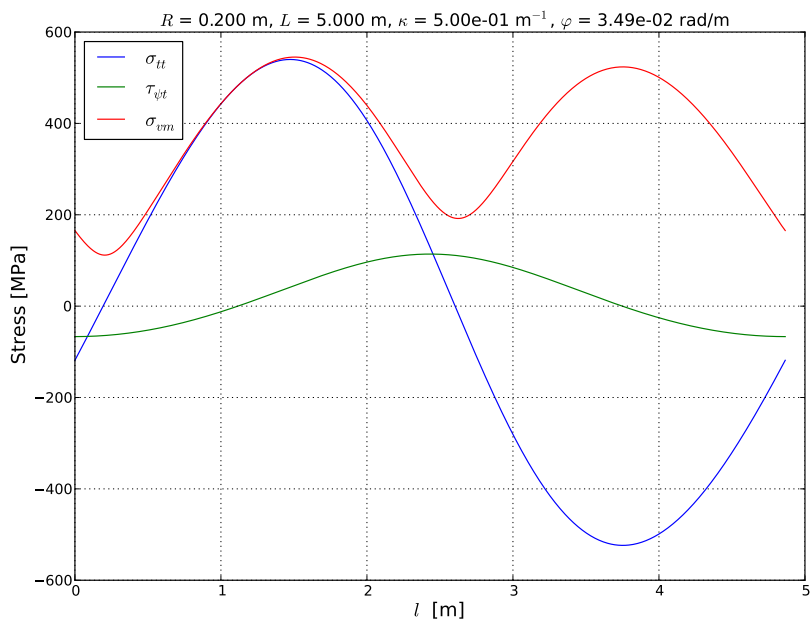

L. Jordal, R. Slora, E. Vermeer, and M. Komperød. A novel bending stiffness rig for identification of subsea cables' and umbilicals' sensitivity to temperature under sinusoidal curvature oscillations. In Proceedings of the Twenty-seventh International Ocean and Polar Engineering Conference (ISOPE 2017), San Francisco, California, USA, 2017.

E. Kebadze. Theoretical modelling of unbonded flexible pipe cross-sections. PhD thesis, South Bank University, 2000.

R. H. Knapp. Derivation of a new stiffness matrix for helically armoured cables considering tension and torsion. International Journal for Numerical Methods in Engineering, 14:515 $-529,1979$.

M. Komperød. Deriving analytical axisymmetric cross section analysis and comparing with FEM simulations. In Proceedings of the 55th Conference on Simulation and Modelling (SIMS 2014), Aalborg, Denmark, 2014.

M. Komperød. Calculating arc length and curvature of helical elements in bent cables and umbilicals using Fourier series. In Proceedings of the 36th International Conference on Ocean, Offshore and Arctic Engineering (OMAE 2017), Trondheim, Norway, 2017. 
M. Komperød, J. I. Juvik, G. Evenset, R. Slora, and L. Jordal. Large-scale tests for identifying the nonlinear, temperaturesensitive, and frequency-sensitive bending stiffness of the NordLink cable. In Proceedings of the 36th International Conference on Ocean, Offshore and Arctic Engineering (OMAE 2017), Trondheim, Norway, 2017.

M. Lutchansky. Axial stress in armor wires of bent submarine cables. Journal of Engineering Industry, 91(3):687 - 693, 1969.

P. Maioli. Bending stiffness of submarine cables. In Proceedings of the 9th International Conference on Insulated Power Cables (Jicable '15), 2015.

A. Pressley. Elementary differential geometry - 2nd edition. Springer-Verlag London Limited, 2012.

N. Sødahl, G. Skeie, O. Steinkjær, and A. J. Kalleklev. Efficient fatigue analysis of helix elements in umbilicals and flexible risers. In Proceedings of the 29th International Conference on Ocean, Offshore and Arctic Engineering (OMAE 2010), Shanghai, China, 2010.

G. Skeie, N. Sødahl, and O. Steinkjer. Efficient fatigue analysis of helix elements in umbilicals and flexible risers: Theory and applications. Journal of Applied Mathematics, 2012.

J. Tarnowski. Improved method of determining bending stiffness of underground cables. In Proceedings of the 9th International Conference on Insulated Power Cables (Jicable '15), 2015.

D. D. Tjahjanto, A. Tyrberg, and J. Mullins. Bending mechanics of cable cores and fillers in a dynamic submarine cable. In Proceedings of the 36th International Conference on Ocean, Offshore and Arctic Engineering (OMAE 2017), Trondheim, Norway, 2017. 